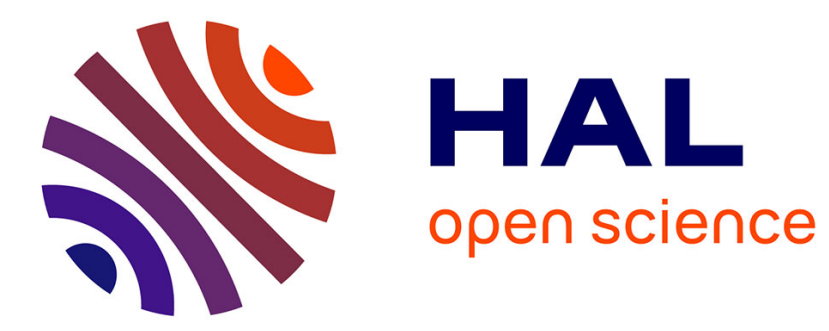

\title{
Les forêts de l'Allemagne an XVIe siècle
}

M. Deveze

\section{To cite this version:}

M. Deveze. Les forêts de l'Allemagne an XVIe siècle. Revue forestière française, 1962, 6, pp.479-493.

$10.4267 / 2042 / 24470$. hal-03386277

\section{HAL Id: hal-03386277 \\ https://hal.science/hal-03386277}

Submitted on 19 Oct 2021

HAL is a multi-disciplinary open access archive for the deposit and dissemination of scientific research documents, whether they are published or not. The documents may come from teaching and research institutions in France or abroad, or from public or private research centers.
L'archive ouverte pluridisciplinaire HAL, est destinée au dépôt et à la diffusion de documents scientifiques de niveau recherche, publiés ou non, émanant des établissements d'enseignement et de recherche français ou étrangers, des laboratoires publics ou privés. 


\title{
LES FORÊTS DE L'ALLEMAGNE AU XVI• SIECLE
}

\author{
PAR \\ Michel DEVĒZEE \\ Professeur à la Faculté des Lettres \\ et des Sciences Humaines de Nancy
}

L'Allemagne est un des pays du monde qui ont le plus aimé la forêt et la chasse. En témoignent encore aujourd'hui l'existence de très vastes forêts bien entretenues et l'organisation d'écoles forestières bien vivantes. L'historien forestier allemand, M. RUBNER, qui a participé à ce cycle de conférences, souhaitait dans un récent article de revue qu'il y eut une tentative de synthèse sur un siècle de l'histoire de l'Allemagne dans le domaine des forêts. Un tel travail n'existe pas Outre-Rhin. Pourtant les études d'histoire forestière sont nombreuses en Allemagne: mais ce sont des travaux sur l'évolution générale de la forêt allemande depuis les temps préhistoriques (œuvres de Schwappach, Roth, BeRnHard, HILF), ou bien ce sont des études régionales approfondies, comme celles de Timm sur les coutumes forestières du Nord-Ouest de l'Allemagne, de Mager sur les forêts de la Prusse Orientale, de von Hornstein sur les forêts des Préalpes ou du plateau Bavarois, de KASPERS sur le "Comté des forêts de la région de Trèves - Aix-la-Chapelle ») ou encore des travaux sur le droit forestier (Professeur MANTEL).

Je vais donc me risquer à présenter cet essai sur " la forêt allemande au $\mathrm{XVI}^{\circ}$ siècle ». Tout d'abord, il me parait profitable de vous présenter brièvement la situation de la forêt française à cette époque, afin de faire d'utiles comparaisons.

La forêt française avait presque atteint, dès le début du XIV ${ }^{\theta}$ siècle, à la veille de la guerre de Cent Ans, ses limites actuelles, tout au moins dans les régions de plaines. La guerre de Cent Ans n'a que peu modifié l'étendue des forêts françaises, mais elle a retardé l'évolution économique de la France, et par là même retardé la crise forestière qui n'éclatera qu'au début $\mathrm{du} \mathrm{xvI}^{\mathrm{e}}$ siècle avec la prospérité revenue, par suite de la croissance de la population 
et de ses besoins tant domestiques qu'industriels. Or, l'Allemagne qui n'a pas connu aux $\mathrm{XIV}^{\mathrm{e}}$ et $\mathrm{XV}^{\boldsymbol{\theta}}$ siècles de luttes aussi meurtrières, se trouve au siècle suivant plus avancée que la France - tout au moins l'Allemagne de l'Ouest et du Sud - dans une évolution économique qui tend au capitalisme, à la commercialisation des bois et à leur transport à longue distance. La France subira avec quelque retard cette évolution qui sera freinée à nouveau à la fin du $\mathrm{XVI}^{\boldsymbol{\theta}}$ siècle par les guerres de religion. De toute manière, ce qu'on appelait l'ancien régime médiéval de la forêt, défini par les coutumiers, qui était libéral pour le paysan, tant pour les droits de pâturage, de chauffage, de bois d'œuvre que pour le droit de chasse, est dans la France du $\mathrm{XvI}^{\mathrm{e}}$ siècle fortement attaqué. Beaucoup de propriétaires forestiers, c'est-à-dire pratiquement le roi, les nobles et les églises cherchent à protéger leur forêt contre ces droits devenus trop lourds par suite de la multiplication des usagers et de leurs bestiaux; ils recherchent une exploitation de la forêt pour eux plus rentable; le désir d'obtenir des profits en argent l'emporte sur l'ancienne habitude de recevoir les redevances en nature des usagers; ils souhaitent vendre leur bois aux marchands et sont donc amenés à réduire non seulement les droits et le nombre des usagers, mais même la portion des forêts sur lesquelles ces droits s'exerçaient. Le triage ou tiercement largement répandu dans le Nord, le Centre et l'Est de la France ne laisse qu'un tiers aux paysans, les deux tiers aux seigneurs. De là des luttes sociales multiples, mais encore localisées. Or, l'Allemagne plus précocement et rapidement engagée dans l'économie moderne connaitra ces luttes avec plus d'acuité encore, à tel point qu'elles dégénéreront en une révolte généralisée des paysans du Sud et de l'Ouest. D'autre part, les forêts françaises diminueront en surface au cours du XVI. siècle (diminution moyenne de 10 à $15 \%$ ), mais seront plus encore atteintes sous le rapport de la qualité; le taillis et les landes se substituant dans mainte région à la futaie. Il s'agit de savoir aussi dans quelle mesure les forêts de l'Allemagne suivant les régions ont subi ces mêmes assauts et y ont résisté.

La forêt en Allemagne est vaste, plus vaste qu'en France, et assez variée. Elle couvre encore actuellement $27 \%$ de la surface du pays (Allemagne de l'Ouest et Allemagne de 1'Est comprises), et $37 \%$ même en Autriche, l'Autriche étant en effet une partie non négligeable de l'Empire allemand de cẹtte époque, le Saint Empire Romain Germanique. $27 \%$ est une forte proportion pour l'Europe, nettement au-dessus de la moyenne. Elle était évidemment plus forte encore au $\mathrm{XVI}^{\mathrm{e}}$ siècle, sans doute de l'ordre de 33 à $35 \%$ (France de l'époque $25 \%$ ). 


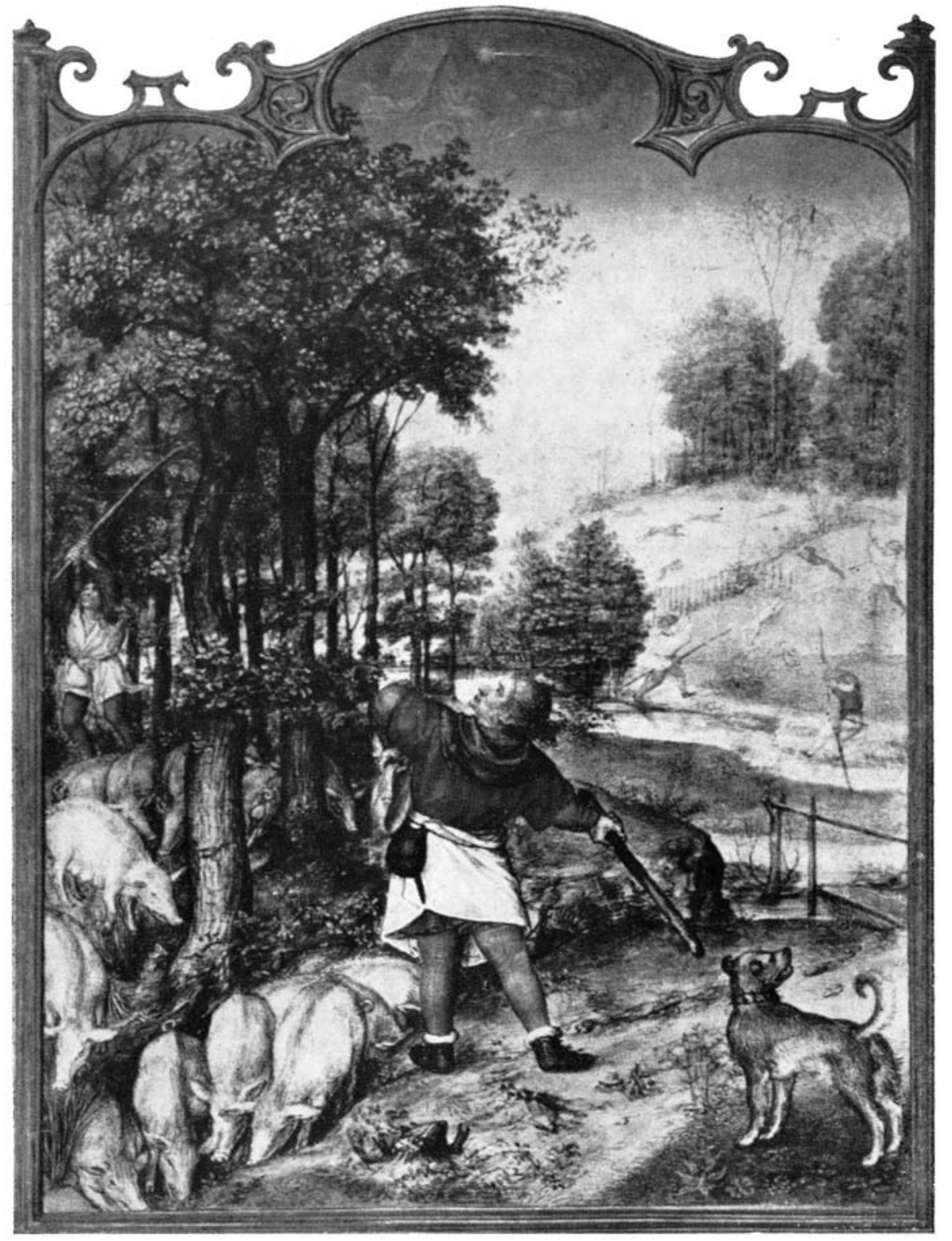

La glandée en forêt (image de Novembre du Brevarium grimani - début du xvI ${ }^{\mathrm{e}}$ siècle). 


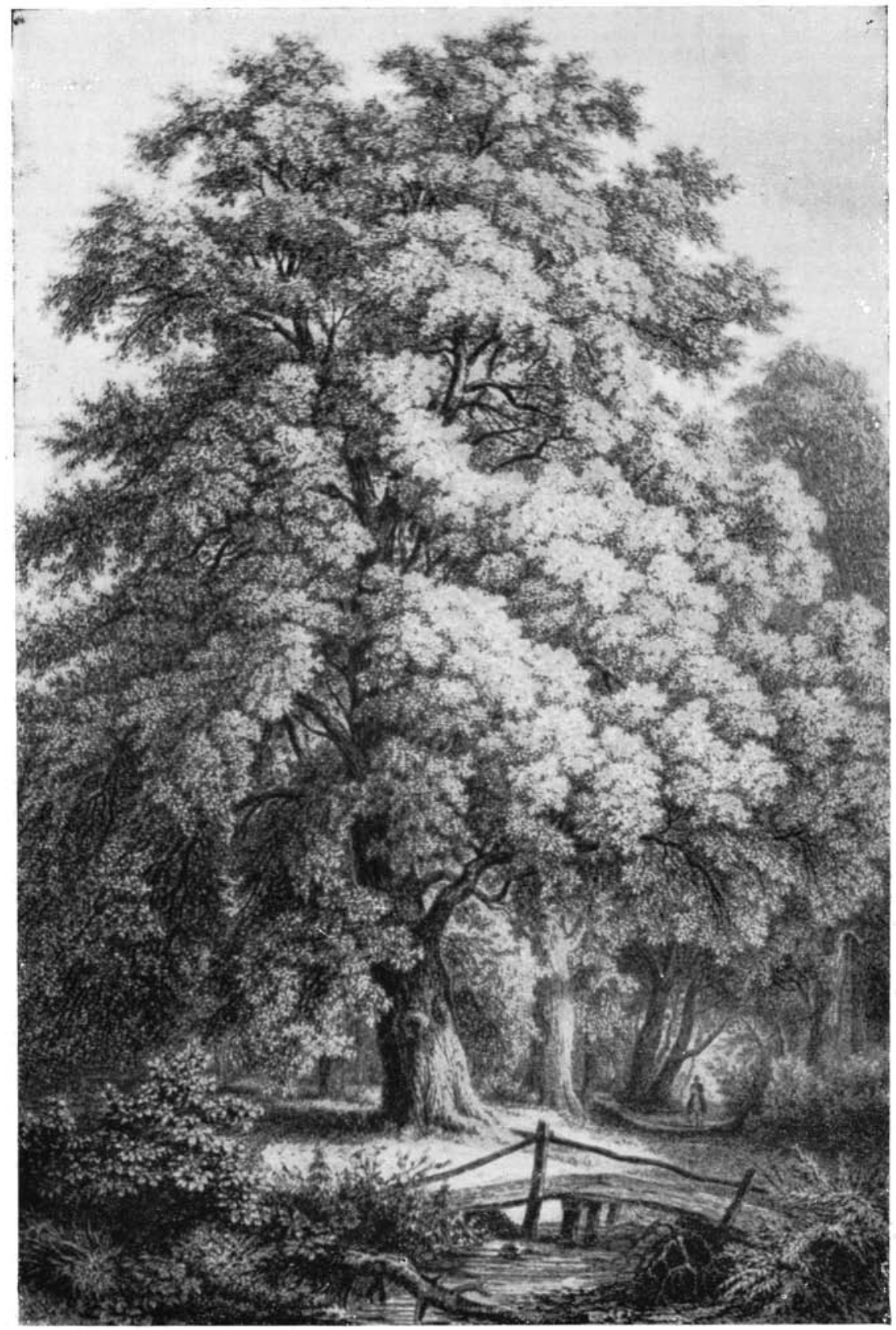

Tilleul (Extrait de Der Wald). 
Les travaux de plusieurs savants: Dengler, Werth, KarL Bertsch, permettent de distinguer plusieurs zones en Allemagne d'après les essences et naturellement en fonction des climats.

$1^{\circ}$ Dans la région la plus voisine de la France (Alsace qui était alors dans 1'Empire, pays de Bade, sauf les sommets des Vosges et de la Forêt. Noire) il y avait essentiellement une forêt mêlée de sapins (Tannenbaum) et de hêtres.

$2^{\circ}$ Au Nord de l'Alsace, dans le Palatinat, le Massif Schisteux Rhénan, l'Allemagne centrale jusqu'à la.Weser, le hêtre était prédominant ; il restait encore beaucoup de futaie, c'était la région où la forêt, qu'on appelait ici la forêt hercynienne, était la plus célèbre dans l'antiquité, elle était encore bien vivante sur les montagnes et les plateaux au $\mathrm{XVI}^{\ominus}$ siècle.

$3^{\circ}$ En Allemagne du Sud, d'une manière générale au Sud du Danube, sur le plateau bavarois, dans les Alpes, sur les sommets également de la Forêt Noire, de l'Odenwald, des Juras Souabe et Franconien, de la Forêt de Thuringe (ce n'est pas un hasard si le mot ( wald: forêt ) veut dire aussi massif montagneux) et encore surtout le pourtour du quadrilatère Bohémien, règne une forêt caractérisée par la présence abondante de l'épicéa ou sapin rouge, qui couvre 30 à $40 \%$ de la surface.

$4^{\circ}$ La Rhénanie Westphalie, au Nord du massif Schisteux Rhénan, est au contraire le pays du chêne (15\% de la surface).

$5^{\circ}$ L'Allemagne du Nord Ouest, en bordure de la mer du Nord, de la Hollande au Danemark, est un pays de landes et de tourbières de climat trop humide et trop venteux pour supporter des forêts (Heidegebiet).

$6^{\circ}$ Un autre pays presque sans forêts est la riche région des limons et des loess (de la Silésie à l'Est à la Weser à l'Ouest), peutêtre $4 \%$ de bois dès le $\mathrm{XvI}^{\ominus}$ siècle.

70 Sur la côte de la mer Baltique, sur les croupes baltiques, la forêt est formée d'un mélange de feuillus (surtout de hêtres et de conifères), (le kiefer, le pin du Nord, $25 \%$ de la surface).

$8^{\circ}$ Plus à l'Est encore, en Prusse Orientale, c'est la zone des Conifères (épicéa, sapin de l'Est), qui s'étend bien au delà dans les pays baltes et en Russie ( 50 à $55 \%$ de la surface).

$9^{\circ}$ Enfin l'Allemagne Centre-Orientale de l'Elbe à la Vistule est également un pays de conifères où prédominent les pins.

Pour résumer, si l'on met à part les móntagnes les plus élevées et les zones dont le sol supporte mal l'arbre, l'Allemagne de l'Ouest et $d u S u d$ est plutôt à l'état naturel la zone des feuillus, l'Allemagne du Noŕd et de l'Est la zone des conifères. 
Les conifères sont plus répandus qu'en France où ils étaient à peine connus au $\mathrm{XVI}^{\mathrm{e}}$ siècle de l'administration royale. Certains historiens du Moyen Age ont même donné à cette division une profonde importance historique: la frontière occidentale d'expansion naturelle du pin Kiefer correspondrait à peu de choses près à la limite d'expansion de l'Empire de Charlemagne qui aurait rejeté les Saxons au delà de la limite des feuillus, les forêts de conifères étant beaucoup moins intéressantes pour le pâturage. Ces différences naturelles subsistent encore presque intégralement dans l'Allemagne du $\mathrm{XVI}^{\ominus}$ siècle.

$\mathrm{Au} \mathrm{XVI}^{\theta}$ siècle, les diverses zones forestières étaient naturellement différemment utilisées. Etudions d'abord ce qui subsiste en Allemagne de l'économie médiévale:

Comme en France, les coutumes (Weistümer) sont encore respectées à peu près partout dans les campagnes. Elle régissent les droits d'usage et de pâturage: le bois est toujours la principale matière prẹmière de l'époque. Comme en France dans les régions de montagnes, il subsiste en Allemagne dans les Alpes, le Bôhmerwald, le Thuringerwald, le massif Rhénan oriental, des forêts de villages: la communauté agraire germanique du Moyen-Age d'esprit collectiviste (la Markgenossenschaft) a survécu dans certains endroits, elle administre ses propres bois, les usagers se servent eux-mêmes dans les communaux. Mais la plupart du temps, la Markgenossenschaft s'est trouvée soumise à un Seigneur qui réglemente de plus en plus sévèrement les droits d'usage, car il est devenu le propriétaire éminent de la forêt. Il exige des redevances croissantes, généralement en avoine.

A relire les vieux textes de coutumes, on constate que le bois est alors le complément nécessaire du champ et du pré, même pour l'alimentation. Il héberge beaucoup de troupeaux de bétail (bovins, chevaux, porcs; et en moindre quantité que dans les pays du Midi, chèvres, ânes et moutons).

La forêt est dite souvent dans les textes : Nährwald: la forêt nourricière. En automne finit dans les forêts de feuillus le pacage des bestiaux. Alors commence ce qu'on appelle en France le panage our la glandée (en allemand: Mast) dont l'essentiel, est constitué par les glands ou les faînes pour la nourriture des porcs. Il n'y a à ce point de vue aucune différence essentielle entre les deux pays. La glandée est généralement réservée aux besoins des animaux du seigneur ou de ceux des villageois. On n'admettait les étrangers que moyennant finance et au cas où la récolte était superbe. L'importance de la glandée, c'est-à-dire le nombre des porcs qu'elle pouvait nourrir, était devenue souvent, comme en forêt d'Hague- 
nau, une mesure de la grandeur, de la valeur même de la forêt. La glandée était d'autant plus importante qu'on ignorait encore à cette époque la pomme de terre. Mais contrairement à l'opinion de certains historiens, je pense que la glandée ne constituait tout de même pas la valeur essentielle de la plupart des forêts de l'Allemagne (même si on ajoute à la glandée ordinaire: glands et faines, ce que les Allenıands appelaient la superglandée (obermast): noix, châtaignes, fruits sauvages, prunelles dont on tirait un alcool, champignons et escargots).

Il est évident que les forêts de conifères étaient à ce point de vue beaucoup moins intéressantes, mais elles possédaient une spécialité: les essaims d'abeilles. L'apiculture a pris un essor en Allemagne Orientale ou dans certaines forêts de l'Allemagne du Sud qu'elle n'a jamais connu au même degré en France. La capture des abeilles, du miel, de la cire, appartenait à l'origine áux libres compagnons de la marche (Freie Markgenossen), c'est-à-dire aux hommes libres de la communauté rurale, mais le développement du système seigneurial avait entraîné au Moyen-Age la création de forestiers spéciaux: les Zeidler, qui dans certaines contrées étaient d'importants personnages, ainsi dans le Fichtelgebirge, la Lusace, la Marche de Brandebourg. Nous connaissons particulièrement les Zeidler royaux des deux forêts proches de Nuremberg (la forêt de Lorenz et celle de Sebald) appelées dans les anciens documents " les jardins d'abeilles du Saint-Empire ». Au xVI siècle, ces forêts étaient devenues propriété de la ville de Nuremberg, mais les Zeidler existaient toujours. Ils étaient fieffés, c'est-à-dire avaient reçu pour prix de leurs services un fief héréditaire de bois. Ils avaient leur propre"tribunal (Zeidelgericht) installé dans un bourg-marché près de Nuremberg, et 27 villages d'apículteurs étaient leurs justiciables. Comme les anciens forestiers royaux, les Zeidler devaient fournir en cas de guerre chacun 6 arbalétriers, ils avaient en échange la franchise des octrois dans toutes les villes libres du Reich. Ces privilèges des Zeidler se comprennent si l'on songe aux services considérables rendus par le miel et la cire jusqu'à l'introduction en Europe de quantités importantes de sucre de canne, c'est-à-dire pratiquement en Allemagne jusqu'à la fin du xvi ${ }^{\mathrm{e}}$ siècle. Il faut penser aussi que l'hydromel (honigwasser) était encore largement en usage. Quant à la cire, elle servait aux tablettes, aux sceaux, à la fabrication des cierges.

A côté de l'apiculture à demi-sauvage dans l'Allemagne de l'Est, une apiculture nouvelle s'était développée en Allemagne de l'Ouest: les abeilles utilisant aussi de préférence les tilleuls et les bruyères. (heidekraut).

Mais il y a une seconde grande utilisation des forêts: ce sont les usages en: bois de chauffage et en bois. d'œuvre. Dans ce domaine, les conifères rendent sans doute plus de service que les feuillus. 
Aussi les Allemands, dès le xive siècle, alors que sont perceptibles les premières difficultés de ravitaillement en bois, chercheront-ils à développer les conifères aux dépens des feuillus.

$\mathrm{Au} \mathrm{XVI}$ siècle, le besoin de confort et l'augmentation de la population accroissaient dangereusement les besoins: en revanche, il y avait une légère diminution de certains usages: par exemple, les établissements de bains, gros consommateurs de bois au MoyenAge, devenaient peu nombreux. Du point de vue de la construction, si les types des formes architecturales n'avaient que peu changé depuis le Moyen Age, il est à noter que l'utilisation des briques, de sable pour les murailles, des tuiles pour les toits augmentait peu à peu au détriment du bois, par suite des dangers considérables d'incendie; ces villes ou villages tout en bois au Moyen-Age flambaient régulièrement tous les 20 ou 30 ans. Les conifères restaient très employés pour le gros œuvre, le pin, le sapin pour les poutres, les solives principales, le mélèze (lärche) pour les portes, les verrous, les volets, même dans les hôtels de ville, les églises. Certes, les rares édifices qui nous restent de cette époque, dans les villes non hombardées au $\mathrm{xx}^{\theta}$ siècle, nous montrent que les édifices les plus aisés n'utilisaient le bois que pour les étages supérieurs, mais la construction en pierre n'était souvent qu'extérieure, toute la charpente, les chevrons du toit étant naturellement en bois.

Parmi les usages du bois assez particuliers à l'Allemagne du Nord, il y avait le revêtement des chemins boueux en planches, le colmatage des trous avec de la ramée, la construction de nombreuses digues en fascines et poutres mêlées. Dans les campagnes, le développement du vignoble exigeait toujours plus d'échalas, souvent en bois d'if, alors qu'en France on les préférait en bois de chêne. La tonnellerie, la tannerie étaient toujours plus exigeantes.

Il subsistait enfin en Allemagne Rhénane. en Souabe et en Franconie, un usage très ancien: l'utilisation du bois pour la construction de parapets en broussailles épaisses et entrelacées, qui rappellent les constructions de défense que les peuplades germaniques de l'antiquité, Chérusques et Angrivariens par exemple, avaient opposées aux Romains. Jusqu'à la guerre de Trente Ans a subsisté ainsi ce qu'on appelait le " gebück du Rheingau ». Large par endroits de plusieurs dizaines de mètres, il formait une véritable fortification autour de la plaine de Bonn. Les arbres étaient coupés à différentes hauteurs, les rejetons bourgeonnants étaient courbés vers le sol; on les y fixait, de nouveaux rejetons sortaient de terre que 'on entrelaçait, le tout renforcé de buissons épineux constituant un mur de protection difficile à franchir. De pareilles fortifications, encore nombreuses à l'époque (il nous reste un protocole de 1619 à leur sujet), devaient être tenues en état par les villages voisins. On appelait normalement ces bocages le Wehrwald, le bois de protection. 


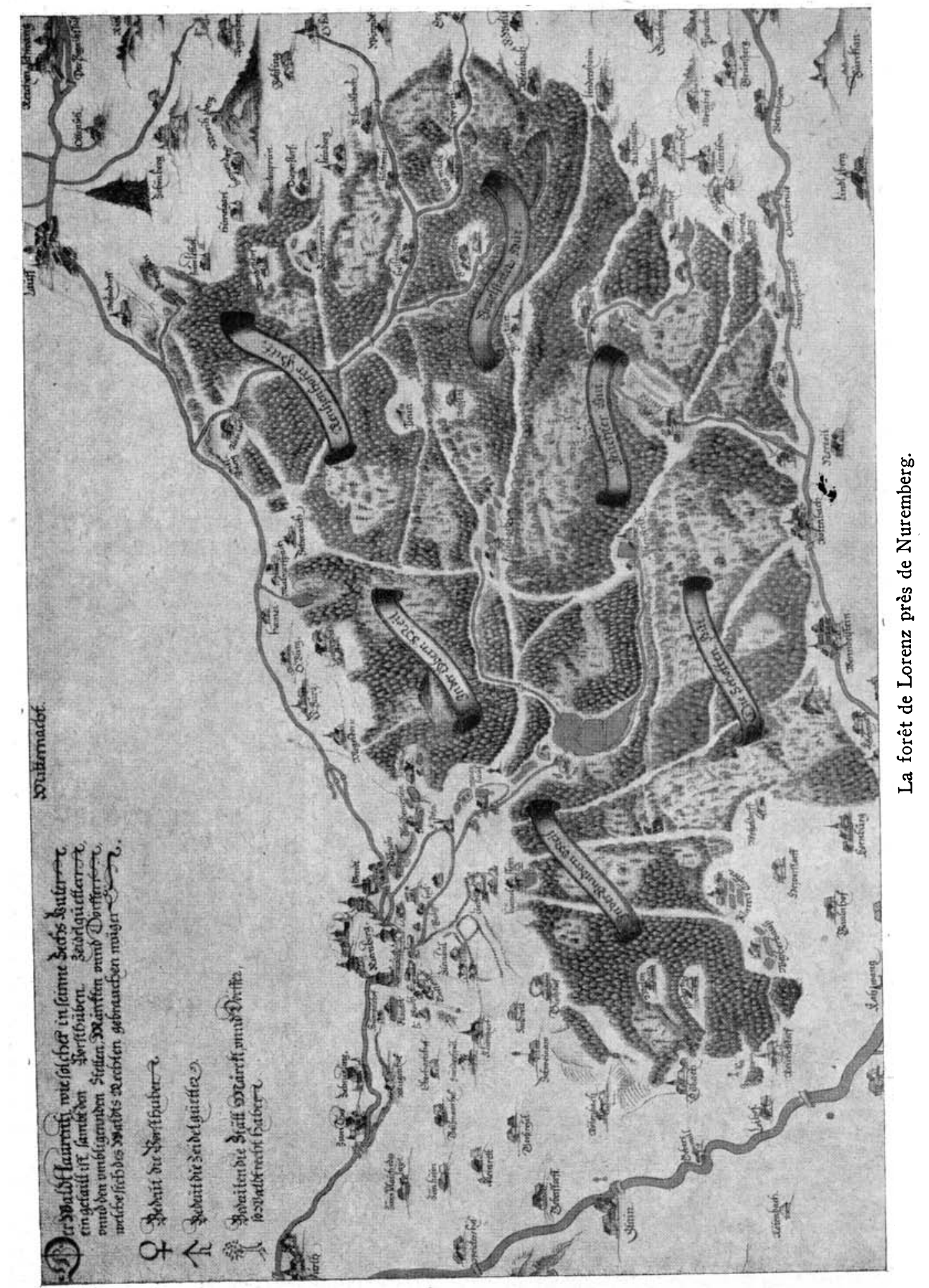




\begin{abstract}
En somme, au $\mathrm{XVI}^{\ominus}$ siècle, le paysage de la forêt allemande différait assez peu de ce qu'elle avait été au $\mathrm{XIV}^{\ominus}$ siècle, époque à laquelle se terminent en Allemagne les grands défrichements, sauf dans quelques montagnes, comme le Spessart, le Harz où ils se continuent même jusqu'au XvI ${ }^{e}$ siècle. Mais depuis le $\mathrm{XIV}^{\mathrm{e}}$ siècle, bien des choses avaient changé sur le plan économique et à côté des vieux usages, une utilisation beaucoup plus commerciale du bois avait commencé. L'Allemagne était le pays de métaux le plus important de l'Europe et avant que l'exploration de l'Amérique n'ait pleinement découvert les trésors du Mexique et du Pérou, c'est-àdire vers 1550 , l'exploitation des minerais de cuivre, de plomb, d'argent et d'or était poussée au maximum, tant en Saxe (Monts Métalliques) que dans le Harz et en Thuringe. D'autre part, le nombre des forges s'était accru au point que sur le plan métallurgique le Reich passait pour le grand fournisseur de l'Europe en armes, outils de fer, objets de quincaillerie, d'orfèvrerie, d'horlogerie, poèles, etc... Les mines de fer du Nassau-Siegen, de Styrie et du Haut Palatinat étaient en pleine prospérité. Les verreries et les salines se développaient aussi, par exemple les salines de Reichenhall dont les ducs de Bavière étaient propriétaires, celles de Hall au Tyrol, dont a récemment parlé M. RuBNER (elles étaient propriété des Habsbourg au $\mathrm{XvI}^{\ominus}$ siècle); les salines du Salzkammergut étaient également propriété des Habsbourg, celles de Salzbourg propriété des archevêques de la ville, celle de Lünebourg en Allemagne du Nord. Toutes les industries, mines, forges, salines, verreries, exigeaient des quantités très considérables de bois. Prenons les salines en exemple: les salines de Lünebourg exigeaient d'après l'historien Bieger $200000 \mathrm{~m}^{3}$ de bois par an, celles de Köstler en Bavière 70000 cordes de bois. Les salines de Hall faisaient venir de très loin du bois par flottage sur l'Inn. En Allemagne du Nord, les salines de Lünebourg étaient alimentées par canaux qui les approvisionnaient en bois depuis le lointain Mecklembourg. En Bavière, le sel de Reichenhall était au contraire transporté vers les forêts de la région de Traunstein, puis quand elles furent épuisées vers Rosenheim.
\end{abstract}

I1 est évident que ces transformations économiques avaient essentiellement profité aux princes, laïques ou ecclésiastiques, quelquefois aux villes propriétaires du sol et du sous-sol, mais aussi aux entrepreneurs, fermiers et exploitants parmi lesquels nous rencontrons au premier rang les capitalistes de la Haute Allemagne: les FugGer qui s'enrichirent dans l'exploitation des mines du Tyrol et de Hongrie, les Welser, les Hochstetter d'Augsbourg, les ImHoF, les HolzschuHER, les FÜRER de Nuremberg; à un moindre degré les marchands de la Hanse en Allemagne du Nord. 
Ces grands capitalistes intervenaient donc forcément dans l'exploitation des forêts allemandes. Nous pouvons citer l'exemple à cet égard de Christoph FÜRER originaire de Nuremberg, anobli sous le nom de Von Haimendorf, né en 1479, mort en 1537. Il avait été représentant à la Diète d'Empire de sa ville natale, conseiller impérial, mais sa grandeur était surtout d'origine économique: spécialisé dans le commerce des métaux, il avait fondé plusieurs usines d'affinage du fer tant en Saxe qu'en Bavière. Il avait encore obtenu un privilège avec son associé Léonard Stockhamer pour le commerce du bois d'if dans les territoires des Habsbourg, et il exportait ce bois très recherché vers la Rhénanie pour la viticulture ou en Angleterre pour la fabrication des arcs. Cette compagnie dura l'espace de 40 ans et exportait la valeur de 20000 arcs par an pour le prix de 30000 florins. Quant à l'autre affaire de Christoph FüRER, continuée par son fils et son petit-fils, celle des forges de Saxe, elle consommait 19000 muids de charbon de bois par an.

Les transports de bois sur les fleuves se multipliaient à cette époque: sans doute le flottage à bûches perdues (wildflosserei on Trift) ou en train était-il très ancien, surtout sur le Rhin et ses affluents. $\mathrm{Au}$ Moyen-Age, le flottage était connu sur l'Inn, sur la Salzach, sur l'Elbe et ses affluents, sur la Vistule. Mais de plus en plus au Xvi siècle on organisait des radeaux de bois, et on transportait de plus en plus le bois en bateau. Le commerce du bois était très important sur le Rhin, la Moselle, la Lahn. C'était, comme en témoignent les estampes, une part essentielle du trafic. Il s'agissait surtout de bois de construction: de grands radeaux de sapins et de feuillus descendaient vers Mayence, Cologne, même la Hollande. Les navires transportaient les planches, la résine, les douves et les cercles de tonneaux. Sur la Moselle, en plus des tonneaux, l'article le plus considérable était le charbon de bois, nécessaire à l'industrie du fer de l'Eifel. Le trafic du Rhin remontait jusqu'aux affluents venus de Forêt Noire: l'association la plus importante de marchands d'eau était la Schifferschaft de la rivière Murg qui a existé jusqu'à nos jours et qui à cette époque exportait jusqu'en Hollande (HolländerHandel).

En Allemagne de l'Est, le port principal du bois était Danżig, où le bois constituait avec ses dérivés (goudrons, résine) un article essentiel du commerce. Les bateaux de la Hanse transportaient beaucoup de bois vers les Pays-Bas, l'Angleterre, le Danemark.

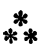

Sur le plan social, il est évident que de telles transformations économiques devaient avoir leur incidence. L'exploitation commerciale des forêts, surtout dans l'Ouest et le Sud de l'Allemagne, contribuait à réduire les droits d'usage. Comme Luther et Melanchthon 
l'avaient prédit avec un certain humour, on manquerait dans peu de temps de trois choses en Allemagne: " de vrais amis, de bonne monnaie et de bois vert $)$.

Les seigneurs étaient de plus en plus chiches à l'égard de leurs vassaux. De plus, ils se mirent à réduire aux habitants des anciennes maisons, construites par exemple depuis un demi-siècle, le bénéfice des droits d'usage et de pâturage. Tous les nouveaux venus, les nouvelles générations habitant les maisons neuves étaient par là même exclus. La réduction des droits forestiers fut donc une des causes, et non des moindres, de la fameuse révolte des paysans qui teinta de sang l'aube du Lutheranisme. Rien d'étonnant que cette réaction défensive des anciens villages communautaires contre l'appropriation individuelle des bois par les seigneurs ait eu pour théâtre l'Allemagne du Sud et de l'Ouest, la plus évoluée au point de vue économique. Il y eut bien sû̀r d'autres causes, qui se rattachent il est vrai au même phénomène de développement du capitalisme. Dès la fin du Xve siècle, il y avait eu des troubles, mais l'occasion de la grande révolte de 1524 fut l'éclatement du Luthéranisme. Les paysans constituèrent une ligue surnommée le Buntschuh, c'està-dire le gros soulier du paysan, et dressèrent un programme en douze articles, qualifiés d'équitables et de fondamentaux, parmi lesquels figuraient en $6^{\circ}$ position une demande de limitation des privilèges seigneuriaux en matière de chasse, et en $8^{e}$ position la demande de restitution des forêts et des pâturages indûment confisqués, à côté d'articles sur les dîmes, les corvées, les banalités, la mainmorte et la remise des dettes.

"Nous sommes lésés en matière de bois, disait textuellement cet article 8, car nos seigneurs se sont tous attribué les forêts et si le pauvre homme a besoin de bois, il doit le payer deux fois plus cher. Et voici quelle est notre opinion:

"les bois que possèdent les autorités, ecclésiastiques on lä̈ques, et qu'ils n'ont jamais achetés doivent faire retour à la communauté tout entière. Et chaque communauté doit avoir ce qu'il lui faut, à chacune ce qui convient pour se chauffer gratuitement; de même le bois de charpente en cas de nécessité avec l'accord de ceux qui seront désignés pour le contrôle par la communauté. Mais si la communauté avait un bien en bois et qu'elle l'ait normalement vendu, alors on doit lui vendre les produits de la forêt en tenant compte des préceptes de l'amour fraternel et des Saintes Ecritures ».

Malheureusement la défaite des paysans en 1525, abandonnés par Luther, eut pour conséquence une dépossession encore plus poussée: des communautés durent pour éviter de plus gros châtiments vendre ou engager les bois qui leur restaient. Cette œuvre de dépossession se poursuivit jusqu'au XVII' siècle. 


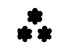

Toutes les conséquences de l'activité capitaliste en forêt ne furent pas aussi fâcheuses; en effet, la technique et la législation forestière firent $\mathrm{au} \mathrm{XVI}^{\mathrm{e}}$ siècle en Allemagne de sérieux progrès, à tel point que l'Allemagne possédait à cette époque une des techniques forestières les plus évoluées de l'Europe. Le technicien le plus connu est Noe Meurer, qui de 1549 à 1583 fut conseiller de l'Electeur palatin et qui rédigea le premier ouvrage réellement technique, bien que pour une large part juridique, qui ait paru en Allemagne et même en Europe concernant les forêts: " Jagd und Forst recht ). (" La chasse et les forêts dans le droit ) (1561, Francfort sur le Main). On peut cependant signaler que la même année 1561 paraissait à Paris en latin " Le commentaire de l'ordonnance forestière de François $I^{\text {er }}$ de 1516 ) de Malleville. Mais Malleville est un pur juriste, Noe Meurer a pu se dégager du droit et raisonner en forestier. En particulier, un récent article du Professeur Mantel, l'actuel directeur de l'Institut Forestier de Fribourg en Brisgau, a par exemple souligné que Noe Meurer a fait campagne, si l'on peut dire, pour l'ensemencement des conifères, dans la seconde édition de son ouvrage signalé plus haut (édition de 1576).

C'est avec l'ensemencement des conifères que débute la technique. moderne en Europe de la reforestation. Le développement des conifères en Allemagne Centrale et Occidentale date de là et atteindra son point culminant au $\mathrm{XIX}^{\boldsymbol{\theta}}$ siècle.

Voici un passage du livre de Meurer:

" il faut récolter les semences de sapin de novembre à mars, les faire sécher dans une chambre, les maintenir dans un endroit sec, pas trop chaud, les enfouir dans le sol en avril lors de la lune descendante au milien de copeaux de sciure, retourner le sol, herser en sous-sol doucement avec une herse de fer... ")
\end{abstract}

Avant Meurer, les recettes techniques en matière forestière étaient de pure tradition. Elles remontaient souvent à une vénérable antiquité (Varron, Caton, Columelle, Palladius), avaient été recopiées fort succinctement dans une compilation médiévale comme celle du Bolonais Betrus de CRescence (vers 1300). En Allemagne, il n'y avait eu jusqu'alors que les calendriers (en allemand: Hausväter; les pères de famille) qui continssent toutes espèces de préceptes mélangeant économie agraire, botanique, élevage, fauconnerie, pêche, chasse. horticulture, médecine et éventuellement, astrologie et explication des rêves. Meurer est le premier à s'être occupé uniquement de culture des bois, sans parler de mythologie ni de divination. A vrai dire, il a récolté lui-même des recettes populaires ou des méthodes déjà reconnues utiles par les forestiers: le professeur Mantel estime qu'il doit son chapitre sur les conifères à des forestiers 
comme un Waldamtmann (Maitre des forêts) anonyme de $\mathrm{Nu}$ remberg: l'ensemencement des conifères, en particulier des sapins, aurait déjà été pratiqué au $\mathrm{xv}^{\ominus}$ siècle dans la région de Nuremberg d'où provenaient les semences qu'on envoyait jusqu'à Francfort où 'se tenait un marché.

Mais Meurer a systématisé ces pratiques, et son influence est notoire sur la littérature forestière de l'avenir, en particulier sur Johannes Colerus, un pasteur du Brandebourg de la fin du $\mathrm{XvI}^{\ominus}$ siècle. Colerus est le second littérateur forestier d'Allemagne chronologiquement: Colerus est l'auteur de deux ouvrages: “Oeconomia oder Hausbuch » (l'économie domestique, dirait-on aujourd'hui), paru à Wittenberg en 1593 et qui eut 4 éditions; l'autre ouvrage est plus spécialement consacré à l'art forestier, il s'intitule: “ Von der Holzung ) et date de 1597: il recommande de semer le Kièfer, d'étendre la culture des conifères, il étudie la meilleure façon de couper le chêne. Il recommande aussi de laisser des étalons en forêt, c'est-à-dire des arbres de réserve, ce que l'on appelle en français des baliveaux. Je ne puis m'empêcher de traduire le conseil sage pour l'époque, qu'il donne en conclusion de son ouvrage pour celui qui veut avancer dans la foresterie (c'est-à-dire la connaissance des forêts):

"Qu'il interroge le bûcheron, le paysan, le défricheur qui s'occupent chaque jour de ces questions, il en tirera une ample connaissance ).

Cependant, Colerus et son prédécesseur Noe Meurer sont moins instructifs en ce qui concerne la législation et les coutumes forestières que les auteurs français de l'époque de Henri IV, ChaufFOURT et SAINT-Yon. La France avait en effet une législation d'ensemble, la législation royale, ce qui s'explique par la prédominance du roi et la centralisation du pays. Le fractionnement de l'Allemagne multipliait les législations régionales, mais aucune n'était encore aussi intéressante que la législation française.

L'organisation forestière de l'Allemagne du $\mathrm{XvI}^{\boldsymbol{\theta}}$ siècle se ressentait de ces divisions. Les forestiers domaniaux des empereurs carolingiens avaient fait place aux nombreux forestiers des princes, des évêques et des couvents, parfois grassement rémunérés et pourvus de fiefs (forestiers fieffés). La victoire des princes territoriaux sur l'administration impériale, encore incertaine du temps de Maximilien d'Autriche, s'est définitivement affirmée grâce aux guerres religieuses nées de la Réforme. Dans les districts industriels où existaient: mines, forges, verreries ou salines, les nécessités du renouvellement. eles approvisionnements en bois contraignaient plus qu'ailleurs à une discipline sévère. C'est dans les régions de salines 
(par exemple au Tyrol, à Salzbourg) que se renforça d'abord une administration forestière hiérarchisée: les forestiers n'étaient plus fieffés, mais viagers et soumis au prince. Ainsi, dans le Tyrol où déjà apparaît au $\mathrm{xv}^{\mathrm{e}}$ siècle un forestier supérieur (obrist-ForstMeister). Depuis 1522, le frère de Charles-Quint, Ferdinand, devenu héritier des possessions des Habsbourg, contrôla lui-même l'administration forestière de ses pays. Ferdinand $I^{\text {er }}$ est connu dans l'histoire de l'Allemagne pour avoir pris un particulier intérêt à l'économie forestière. Ce qui le démontre, ce sont les nombreuses mesures de législation, les instructions qu'il envoya aux forestiers lors de leur installation soit dans le Tyrol, soit dans les autres pays autrichiens (par exemple en Haute Alsace, à Bregenz le long du lac de Constance, dans le Vorarlberg, en Styrie).

L'année 1557 est une année, décisive dans l'histoire forestière des états autrichiens: c'est l'année où parut une ordonnance forestière complète (signée à Prague par Ferdinand le 17 avril). Ferdinand $\mathrm{I}^{\text {er }}$ avait alors succédé à Charles Quint comme Empereur depuis un an. Ferdinand nommait un Königlich-Kaiserlich Forstmeister, l'équivalent du souverain maître des eaux et forêts en France, mais seulement pour les pays héréditaires, non pour tout l'Empire. Le premier titulaire de la fonction, Hans von ANDLAU, était d'une très ancienne famille d'Alsace: il était lui-même déjà d'un certain âge et avait jusqu'alors le titre de Conseiller impérial et Chevalier du Saint-Empire "Sacri romani imperii eques et imperatoris Ferdinandi primi consiliarius intimus "); il avait participé au gouvernement des territoires de l'Alsace, Sundgau. Brisgau, Schwarzwald, extérieurs à l'Autriche elle-même. Il avait aussi exercé la maitrise forestière de la forêt de la Hardt en Haute Alsace.

Il mourut sans doute en 1572. Il existe un portrait de cet Hans Von Andlau qui se trouve aujourd'hui en France, je ne sais à la suite de quelle péripétie, au château de Keranroux, par Ploujean (Finistère). Son successeur fut son fils Johann von ANdLAU; à la fin du siècle ce fut un certain Hans Christoph von Stadion. Le grand forestier touchait alors 200 gulden, ce qui était une somme élevée. Mais les grands forestiers français étaient comparativement mieux rémunérés encore.

Une organisation similaire était adoptée dans la plupart des principautés allemandes assez importantes et disposant de forêts. Les ordonnances forestières se multiplient au $\mathrm{XVI}^{\mathrm{e}}$ siècle, dans le Würtemberg en 1514, pour la principauté d'Anspach en 1531, à Salzbourg en 1524, pour la Hesse en 1532, le Brunswick Wolfenbuttel en 1547, la nouvelle marche de Brandebourg en 1547, l'évêché d'Augsbourg en 1553, le duché de Bavière èn 1568 (il y avait eu déjà de petites ordonnances en Bavière en 1476, 1491, 1536). Dans la seconde moitié du Xvie siècle, on peut encore citer la Saxe électorale, le Palatinat, le margraviat de Baden Baden, le margraviat 
de Bayreuth; les ordonnances se ressemblent d'ailleurs beaucoup: la protection de la chasse, passion des seigneurs est un des motifs le plus sérieux de leur adoption, tout autant que l'intérêt fiscal des princes et le souci de favoriser l'exploitation économique. Sans être aussi perfectionnées que les ordonnances royales françaises de $1516,1517,1518,1543$ et 1583 , les ordonnances sont souvent indigestes, ce qui faisait dire à un conseiller à la cour du Palatin qui avait écouté un dimanche la lecture d'une ordonnance:

$$
\begin{aligned}
& \text { Ach, lieber Gott, } \\
& \text { Wie viel neuer Gebot. } \\
& \text { Las es in Himmel gehen } \\
& \text { Wer kann Sie alle halten. }
\end{aligned}
$$

Ah! Dieu bien aimé,

Que de nouveaux commandements.

Il ira sûrement au ciel

Celui qui pourra tous les retenir!

Dans les ordonnances de la fin du $\mathrm{XvI}^{\mathrm{e}}$ siècle, on trouve aussi quelques préceptes techniques à côté de nombreux paragraphes administratifs et financiers (conservation des graines d'arbres, terrains et climats convenables aux différentes espèces, reforestation des places vides, rajeunissement naturel et artificiel, coupes de bois, etc...). Incontestablement jusqu'à la guerre de Trente Ans, l'art forestier allemand avait fait de très nets progrès. Le mesurage, l'arpentage des forêts s'améliorèrent aussi: on ne mesurait plus les bois d'après le nombre d'animaux qui pouvaient $y$ paițre; en 1590 , le conseiller de la ville de Nuremberg, Paulus Pfinzing mesura toute l'étendue des forêts de la ville avec un compteur de roues d'une voiture et une boussole. Dès 1544 , les bois de Weimar étaient mesurés, ceux de la Thuringe en 1557. On a donc conservé de cette époque des cartes détaillées et souvent artistiques: ainsi, dans ce qu'on est convenu d'appeler l'Atlas Pfinzing, les districts de la forêt étaient peints de couleur diffẹrente. Les frontières de la forêt, chemins, étangs, cours d'eau, enclaves des champs, landes, bruyères sont exactement mesurés et bien visibles (En France, on dispose d'une tentative analogue pour les forêts royales qui réussira tout au moins pour les forêts Normandes en 1564, 1568).

Les cartes régionales apparaissent aussi avec des taches forestières plus ou moins exactes : ainsi la carte de la Prusse par Kaspar HenNeberger, carte très soigneusement étudiée 'pour l'époque.

On connait enfin un certain nombre d'instructions forestières de détail, généralement prises après une visite de la forêt par l'autorité forestière supérieure. Leur étude permet de reconstituer, comme l'ont fait divers auteurs allemands modernes, les premiers aménagements forestiers. 
C'est ainsi qu'on connaît en Allemagne du Sud-Ouest dès le $\mathrm{xv}^{\mathbf{0}}$ siècle, les coupes d'éclaircie (à Haguenau, à Francfort, à Fribourg). Les réformations d'une forêt pour l'aménagement datent de 1510 à Augsbourg (forêt de Wallwarth), le taillis sous futaie (Mittelwald) y est pratiqué et de là, la pratique émigre à Wurzbourg en 1575 ; le taillis sous futaie est également connu à Memmingen (Bavière méridionale) en 1570; il est recommandé par le Holzmeister Schwefelin.

La guerre de Trente Ans fut le début d'une nouvelle période dans l'histoire des forêts de l'Allemagne. Cette époque, si terrible pour la plus grande partie du pays, entraîna une gestion désastreuse des forêts, une exploitation désordonnée, un recul des méthodes d'aménagement. Quelle différence'avec le $\mathrm{XVI}^{\mathrm{e}}$ siècle qui, malgré les luttes religieuses, avait été une des périodes les plus prospères de l'histoire du peuple allemand! L'Allemagne au $\mathrm{XvI}^{\mathrm{\theta}}$ siècle avait su conserver ses massifs forestiers, malgré une exploitation de plus en plus poussée, grâce à un aménagement déjà rationnel. Le développement de la population, l'activité minière et aussi les facilités de flottage et de transport sur les principaux cours d'eau avaient alors entrainé de façon irrésistible la forêt allemande dans le cycle économique moderne. Il faudra attendre la seconde moitié du XviII ${ }^{\circ}$ siècle pour retrouver pareille prospérité et assister à une amélioration des méthodes d'aménagement de la forêt allemande. 